\title{
Cirugía del incidentaloma suprarrenal
}

\author{
Dres. Javier Chinelli, Gustavo Rodríguez ${ }^{\dagger}$ \\ Clínica Quirúrgica 2, Hospital Maciel. Corporación Médica de Canelones (COMECA)
}

\section{Resumen}

Introducción: la incidencia de incidentalomas suprarrenales ha aumentado con el uso de técnicas modernas de imagen y la indicación quirúrgica se basa en la sospecha de malignidad o hiperfunción.

Objetivos: revisar una casuística de incidentalomas suprarrenales operados.

Pacientes y método: 16 pacientes operados entre los años 2010 y 2017 en dos instituciones de asistencia médica. Se describen aspectos diagnósticos, técnica operatoria y morbimortalidad de los procedimientos.

Resultados: la mayoría de los procedimientos se realizaron por abordaje laparoscópico, destacándose tres complicaciones intraoperatorias, sin mortalidad en la serie. La lesión más frecuentemente encontrada fue el adenoma no funcionante.

Discusión: la llave diagnóstica, clave en la decisión de seguir u operar un incidentaloma suprarrenal, es la imagenología. El protocolo de seguimiento continúa siendo objeto de debate actualmente, así como la indicación de cirugía en lesiones funcionantes subclínicas.

Conclusiones: la suprarrenalectomía laparoscópica es una cirugía segura, con cifras de morbimortalidad en el rango descrito por la literatura.

Palabras clave: INCIDENTALOMA SUPRARRENAL

Key words: $\quad$ ADRENAL INCIDENTALOMA

\section{Introducción}

Un incidentaloma suprarrenal (IS) es una masa mayor de $1 \mathrm{~cm}$ de diámetro descubierta en forma inesperada en una prueba de imagen realizada en un paciente sin sospecha de enfermedad a nivel de la glándula. Se trata de una situación relativamente frecuente, estimándose una prevalencia de $5 \%$ a $6 \%$ de la población, cifra que varía según la edad del paciente: menor a $1 \%$ antes de los 30 años; $3 \%$ a los 50 años, y mayor a 15\% después de los 70 años ${ }^{(1)}$. Son bilaterales en $10 \%$ a $15 \%$ de los casos. Con respecto a la etiología, la mayoría tienen origen en la corteza suprarrenal, menos frecuentemente en la médula, y raramente se trata de lesiones de origen mesenquimal o de metástasis suprarrenales $^{(2,3)}$. Casi $10 \%$ corresponde a lesiones no neoplásicas como quistes, pseudoquistes, hematomas e infecciones granulomatosas. Sin embargo, entre todas estas posibles etiologías debemos hacernos dos preguntas que guiarán nuestro análisis y futura conducta terapéutica: si estamos ante una lesión funcionante y si se trata de una lesión sospechosa de malignidad. La imagenología juega un rol fundamental y es la llave diagnóstica en la mayoría de los pacientes, siendo clave junto a los estudios humorales para definir la conducta a seguir: observación versus resección quirúrgica. Las indicaciones de cirugía están claramente establecidas: lesiones secretantes (hipercortisolismo, hiperaldosteronismo, feocromocitoma) independientemente del tamaño y tumores con sospecha de malignidad ( $>4 \mathrm{~cm}$, invasión local, densidad mayor a $20 \mathrm{UH})$. Asimismo, existen actualmente dos puntos de mayor controversia: uno en relación con la indicación quirúrgica de lesiones con secreción subclínica de cortisol, y otro en cuanto al costo/beneficio de los protocolos actuales de seguimiento.

\footnotetext{
* Asistente de Clínica Quirúrgica 2.

† Profesor Titular de Clínica Quirúrgica 2.

Correspondencia: Dr. Javier Chinelli. Mercedes 1472/402, Montevideo, Uruguay. Correo electrónico: jchinelli01@hotmail.com

Los autores declaran no tener conflicto de intereses.

Recibido: 10/7/17

Aprobado: 14/8/17
} 


\begin{tabular}{|ll|}
\hline Tabla 1. Clasificación de Clavien-Dindo* \\
\hline Grado & \\
\hline I & Desviación del posoperatorio normal, que no requiere de tratamiento farmacológico, quirúrgico, endoscópico o intervenciones radiológicas \\
\hline II & Fármacos permitidos: antieméticos, antipiréticos, analgésicos, diuréticos, electrolitos y fisioterapia \\
\hline III & Complicación que requiere una intervención quirúrgica endoscópica o radiológica de tratamiento farmacológico con drogas diferentes a las permitidas en las complicaciones grado I \\
IIIla & No requiere anestesia general \\
IIIb & Requiere anestesia general \\
\hline IV & Complicación que amenaza la vida del paciente y que requiere de cuidados intensivos \\
IVa & Disfunción de un órgano (incluye diálisis) \\
IVb & Falla multiorgánica \\
\hline V & Muerte del paciente \\
\hline
\end{tabular}

El objetivo de este trabajo es revisar una casuística de pacientes con diagnóstico de IS en los que se realizó la adrenalectomía, en particular, indicación quirúrgica, tratamiento efectuado, complicaciones y mortalidad.

\section{Pacientes y método}

Se trata de un estudio de carácter retrospectivo, observacional y descriptivo. La serie consta de 16 pacientes asistidos en dos instituciones: Hospital Maciel y Corporación Médica de Canelones (COMECA) entre los años 2010 y 2017. Se incluyeron todos aquellos pacientes a quienes se les realizó una adrenalectomía con diagnóstico previo de IS.

Para cada caso se consignó edad, sexo, tamaño de la lesión, método imagenológico de detección y motivos por los que fue indicado, estudios de valoración funcional cortical y medular (cortisol plasmático, prueba de supresión de dexametasona, aldosterona, metanefrinas y ácido vanililmandélico en orina), vía de abordaje quirúrgico (convencional o laparoscópica), duración del procedimiento, estadía hospitalaria posoperatoria, anatomía patológica definitiva y complicaciones, utilizando para las últimas la clasificación de Clavien-Dindo (tabla 1). La información fue obtenida a partir de las historias clínicas de los pacientes y se recogió en una planilla Excel para su posterior análisis.

Con respecto a la técnica quirúrgica utilizada ${ }^{(4)}$, particularmente en el caso del abordaje laparoscópico transperitoneal, se coloca al paciente en decúbito lateral con cambrado para aumentar la distancia costo-ilíaca y así obtener mejor exposición. El cirujano y el primer ayudante frente al paciente, el segundo ayudante del otro lado, instrumentista al lado del cirujano. Se emplaza un trócar de $10 \mathrm{~mm}$ para la cámara, algo por encima del ombligo en la línea medioclavicular, luego otros dos de 10 $\mathrm{mm}$ debajo del reborde costal en la línea axilar anterior y media, siendo opcional un cuarto trócar subxifoideo para retracción. La exposición durante la suprarrenalectomía izquierda se realiza liberando el ángulo esplénico del colon, así como la cara externa del bazo, reclinándolos hacia la línea media junto a la cola del páncreas, se clipa la vena capsular media y se secciona, y se sellan los ramos arteriales superiores, medios e inferiores, disecando la glándula desde el tejido adiposo periglandular. Para la suprarrenalectomía derecha la exposición implica la sección del ligamento triangular reclinando el hígado en sentido medial hasta visualizar la vena cava inferior, pudiendo ser necesario el descenso del ángulo hepático del colon, y la sección del peritoneo posterior para acceder a la fascia de Gerota. El resto de los pasos técnicos son similares que los descritos para la adrenalectomía izquierda, destacando que el punto crítico es la identificación, clipado y sección de la vena capsular media, que desemboca directamente en el sector posterolateral derecho de la vena cava inferior. En todos los casos se extrae la pieza en bolsa a través de una minilaparotomía ampliando alguno de los abordajes para los trócares.

\section{Resultados}

La edad promedio de los pacientes fue de 58 años, con un rango que fue desde 37 a 77 años. 

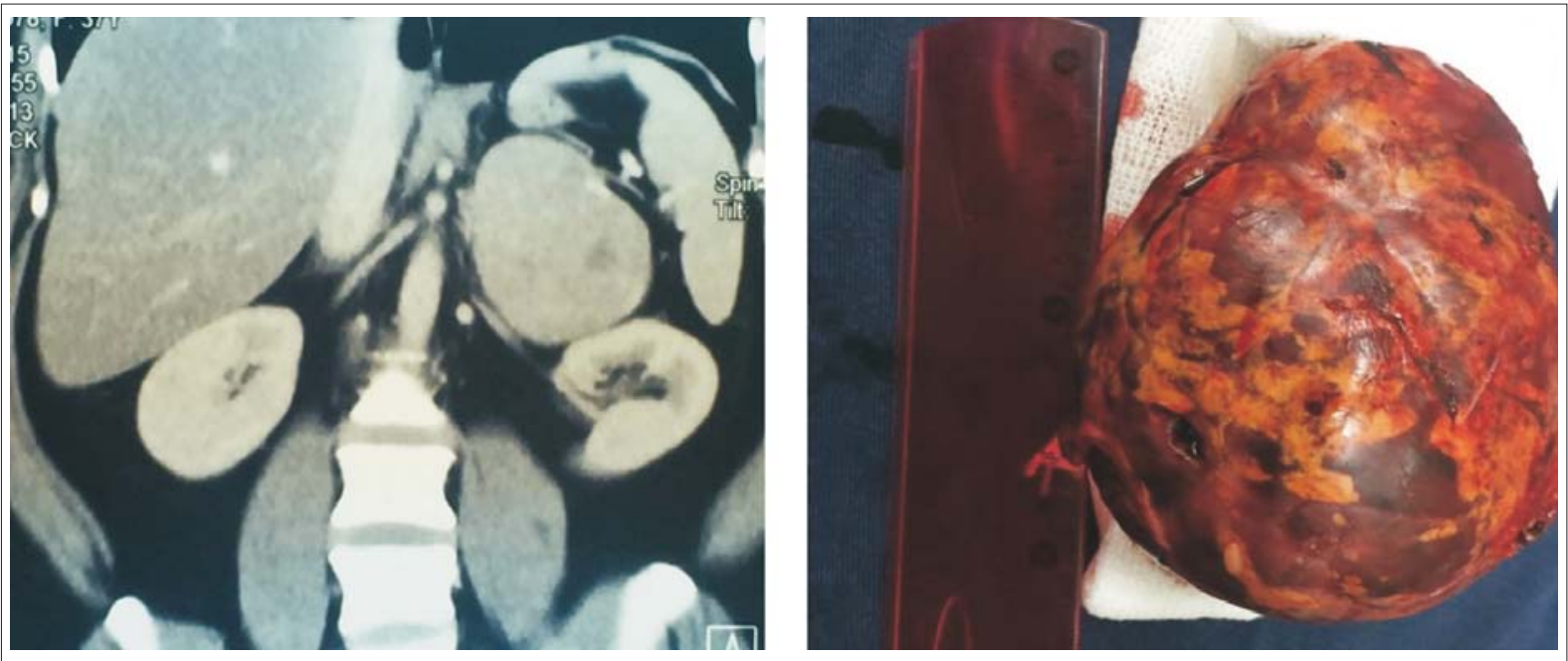

Figura 1. Tumor suprarrenal izquierdo.
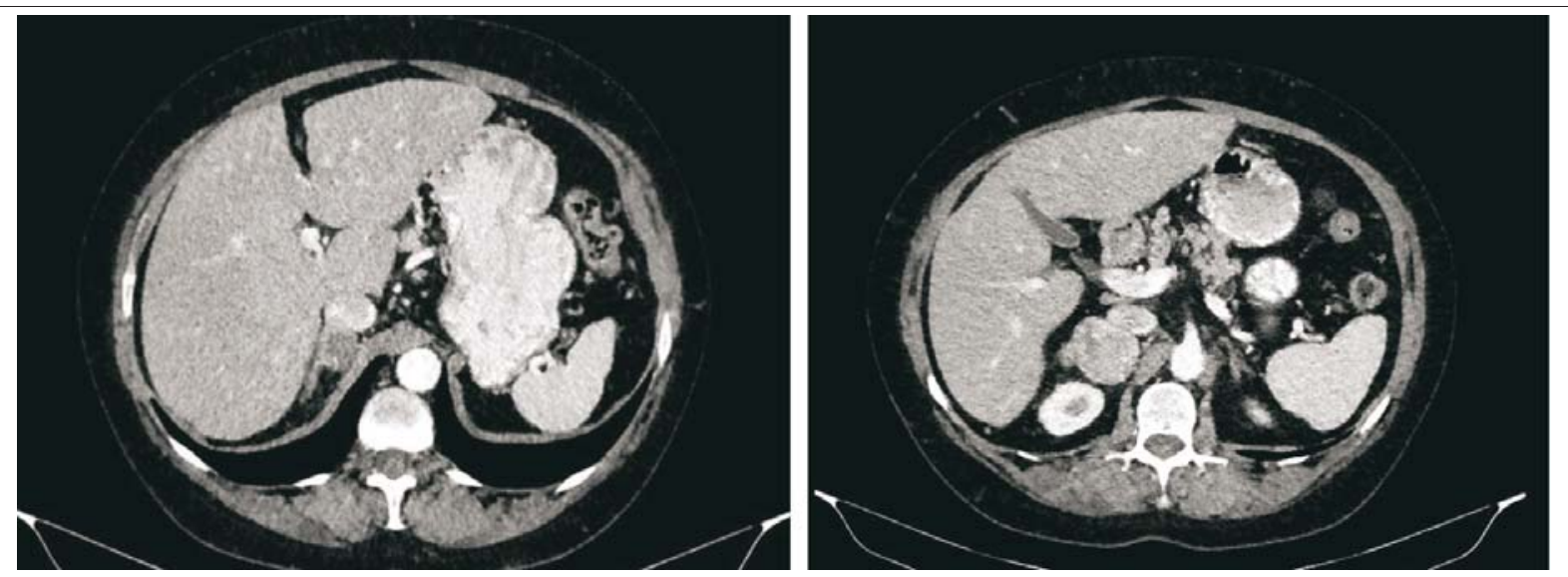

Figura 2 Tumor suprarrenal derecho. Relación con la vena cava inferior.

Once pacientes eran de sexo femenino y cinco masculino.

En todos los casos se trataba de lesiones unilaterales, con un tamaño promedio de $6 \mathrm{~cm}$ (rango $2-12 \mathrm{~cm}$ ).

En todos los pacientes se realizó una tomografía computada como método imagenológico para orientación diagnóstica, destacando que en la gran mayoría de ellos fue la TAC el estudio inicial en el que se identificó la lesión suprarrenal por primera vez, por lo que no fue necesario repetirla. En dos pacientes no fue la tomografía el estudio que detectó la imagen suprarrenal, tratándose en un caso de un politraumatizado donde la ecografía abdominal reveló la masa adrenal, y en el otro una enterorresonancia magnética realizada a una paciente con enfermedad celíaca. A excepción de estos dos pacientes y de otros dos en los que se solicitó el estudio por la presencia de litiasis renal (cólico nefrítico), en los 12 restantes el motivo de solicitud de imagenología abdominal fue la presencia de dolor abdominal inespecífico.

Dos pacientes presentaron lesiones secretantes de cortisol, sin claros elementos clínicos de hipercortisolismo (síndrome de Cushing). Si bien para establecer la presencia de hipersecreción/hiperfunción nos basamos en un resultado de laboratorio alterado, se destaca también el caso de una paciente joven con hipertensión arterial (HTA) severa y estudios de función medular normales, en que la anatomía patológica definitiva reveló la presencia de un feocromocitoma, y cuya sintomatología retrocedió luego de la adrenalectomía. Podemos concluir en este caso que pese a los estudios de laboratorio normales, se trataba de una lesión funcionante.

Por lo anteriormente descrito, en tres pacientes la indicación de cirugía se basó en la presencia de hiperfunción, en tanto que en los 13 restantes la indicación qui- 
rúrgica se debió fundamentalmente al tamaño de la masa.

En cuanto a la vía de abordaje, en dos casos fue laparotómica. En los 14 restantes fue laparoscópica.

El tiempo de duración promedio de las cirugías fue de 117 minutos (rango 35-200 min).

La estadía hospitalaria posoperatoria fue de 72 horas (3 días) en promedio.

El estudio anatomopatológico final de las lesiones resecadas determinó la presencia de nueve adenomas, cinco feocromocitomas, un mielolipoma y un hematoma.

Se registraron las siguientes complicaciones, todas clasificadas como grado III siguiendo la clasificación de Clavien: una lesión duodenal (en cirugía laparotómica) detectada y reparada en el intraoperatorio, con buena evolución posterior; un sangrado intraoperatorio de la logia suprarrenal; una lesión esplénica que requirió la esplenectomía también por vía laparoscópica. No se registraron muertes a causa de estas complicaciones.

En nuestra serie observamos resultados similares a los señalados en la bibliografía, con un buen rendimiento diagnóstico de la tomografía para determinar la naturaleza del IS, a excepción de dos casos: en uno, más allá de que la lesión superaba los $6 \mathrm{~cm}$, no fue posible afirmar que se trataba de un adenoma por no presentar características típicas. En el otro caso (paciente hemofílico) la lesión, que tampoco tenía elementos distintivos, resultó ser un hematoma suprarrenal. El porcentaje de lesiones funcionantes es similar al referido en la literatura $(19 \%)$, y la principal indicación de cirugía sigue siendo el tamaño y, en consecuencia, la necesidad de descartar malignidad. Asimismo, la mayoría de los IS correspondía a adenomas no funcionantes corticosuprarrenales.

\section{Discusión}

El IS es una entidad cuya frecuencia ha tenido un aumento relativo producto del uso cada vez más frecuente de estudios imagenológicos de alta resolución y al envejecimiento progresivo de la población. Dado que las dos principales indicaciones de cirugía son la hiperfunción (presente solamente en $15 \%$ de los incidentalomas) y la sospecha de malignidad, o más frecuentemente la imposibilidad de excluirla por completo, los estudios de función cortical y medular, así como la imagenología, son los principales pilares diagnósticos sobre los cuales descansa la decisión de optar por la cirugía o bien la observación y el seguimiento. Se destaca como elemento predictor de malignidad el tamaño de las lesiones, con un riesgo menor a $2 \%$ en lesiones $<2 \mathrm{~cm}$, pero que asciende a $25 \%$ en aquellas $>6 \mathrm{~cm}^{(5)}$.
Con respecto a la imagenología, constituye la llave diagnóstica, contando con estudios morfológicos (tomografía y resonancia magnética) y funcionales (centellograma y PET-scan). La tomografía computada utilizando cortes finos va a determinar el tamaño de la lesión y sus relaciones con estructuras y órganos adyacentes. Actualmente se considera de mayor utilidad el protocolo cuadrifásico $^{(6)}$ (fases sin contraste, arterial, portal y tardía a los 5 minutos). Interesan tres criterios fundamentalmente: el tamaño de la lesión, la densidad sin contraste, y el realce tardío a los 10-15 min luego de la inyección de contraste para calcular el wash-out absoluto y relativo. En la fase sin contraste, la densidad ayuda a distinguir lesiones benignas (con alto contenido graso en más del $70 \%$ de los casos), de las malignas, con una sensibilidad y especificidad respectivas de $71 \%$ y $98 \%$. Se establece un umbral de 10 unidades Hounsfield (UH) por debajo del cual es casi una certeza que se trate de lesiones benignas, aunque se acepta también de un coeficiente de atenuación de $20 \mathrm{UH}$ o menor. El comportamiento de la lesión tras la inyección de contraste resulta de utilidad sobre todo en aquellos adenomas pobres en contenido graso (30\%-50\% del total, densidad $>10$ UH). A partir de esto se calculan los porcentajes de wash-out absoluto y relativo, que son idependientes del contenido lipídico de la lesión. Valores mínimos de reducción del wash-out relativo y absoluto de $40 \%$ y $60 \%$, respectivamente, permiten hacer diagnóstico de adenoma con una especificidad de $100 \%$. Los tumores malignos, y en menor medida los feocromocitomas, tienen por lo general una densidad $>10 \mathrm{UH}$, son heterogéneos e hipervasculares, y presentan un wash-out mucho menor en fase tardía. Los hematomas son espontáneamente hiperdensos sin contraste, los mielolipomas son hipodensos y los quistes son masas redondeadas de paredes finas que no realzan con contraste. En nuestra serie fue clave el estudio imagenológico en la totalidad de los pacientes, aportando datos fundamentales en cuanto al tamaño y aspecto, determinantes en la mayoría de los casos de la indicación quirúrgica.

La resonancia nuclear magnética no aporta datos adicionales a los de la TAC, por lo que podría ser de utilidad en los pacientes que no pueden recibir medio de contraste yodado ${ }^{(7,8)}$.

El centellograma con meta-yodo-bencil-guanidina es un estudio de excelente rendimiento para el diagnóstico de feocromocitoma pero de difícil implementación dada la prolongada duración del mismo (48 horas).

El PET-scan permite un estudio simultáneo y combinado, imagenológico, y de la actividad metabólica de la glándula ${ }^{(9)}$. La captación del trazador puede estimarse en forma visual y cuantitativa mediante el Standard Uptake Value (SUV), en caso de que el cociente entre el 
SUV del incidentaloma/SUV del hígado sea $>1,45$ es altamente predictivo de malignidad ${ }^{(10)}$, en cuyo caso emergen principalmente cuatro diagnósticos: feocromocitoma, carcinoma adrenocortical, metástasis y linfoma.

Con respecto a la punción biópsica con aguja fina (PAAF), su principal indicación sería ante la sospecha de metástasis suprarrenal y solamente si el resultado de esta modificara la conducta terapéutica ${ }^{(11)}$. Por otra parte, tiene muy bajo rendimiento para distinguir entre un adenoma y un carcinoma suprarrenal. En todos los casos deberá descartarse previamente que se trate de un feocromocitoma, ya que la punción puede precipitar una crisis adrenérgica.

Actualmente, la vía de abordaje quirúrgico de elección para realizar la adrenalectomía es la laparoscópica, que en nuestra serie se realizó en el $88 \%$ de los casos. Su principal limitante es el tamaño de la lesión, si bien pueden llegar a resecarse tumores de hasta $10 \mathrm{~cm}$ dependiendo de la experiencia del equipo quirúrgico. Otra limitante es la presencia de invasión de estructuras vecinas, en cuyo caso se prefiere un abordaje abierto de inicio. La sospecha de malignidad no contraindica de por sí el abordaje miniinvasivo, debiendo tener la precaución de evitar la disrupción capsular. La táctica debe ser la realización de una suprarrenalectomía, no aceptándose resecciones parciales. Las ventajas del abordaje laparoscópico son varias, entre ellas las comunes al carácter miniinvasivo (menos dolor y necesidad de analgesia posoperatoria, alta precoz, rápida inserción laboral, complicaciones parietales). La posición del paciente es en decúbito lateral, con lo que aquellas estructuras intraperitoneales móviles (colon, bazo) caen por acción de la gravedad. El abordaje retroperitoneal (posterior) surge como una alternativa al anteriomente descrito. Si bien evita la apertura del peritoneo, lo que podría resultar ventajoso en caso de laparotomías previas que hagan suponer la formación de adherencias (si bien esto no contraindica en absoluto el abordaje laparoscópico), tiene como desventaja el campo de trabajo reducido y la mayor dificultad para la visualización y el control de las estructuras vasculares. Su principal indicación es la suprarrenalectomía bilateral (por hiperplasia nodular bilateral o feocromocitoma bilateral), no requiriendo cambios de posición del paciente como ventaja adicional. El equipo anestésico deberá prestar especial atención a posibles cambios hemodinámicos o metabólicos intraoperatorios durante la resección de un feocromocitoma, así como de una crisis addisoniana posoperatoria en el caso de los adenomas secretores de cortisol, esta última se previene mediante el aporte exógeno de corticoides que se irá reduciendo paulatinamente.
Hay dos puntos sobre los que existe actualmente controversia. El primero, respecto de los adenomas con hipersecreción subclínica de cortisol, es decir aquellos con secreción autónoma (no suprimida por dexametasona), pero no suficiente como para que se exprese clínicamente un síndrome de Cushing. Se estima que el riesgo de progresión anual a un síndrome clínico es de $12,5 \%{ }^{(12)}$. En estos pacientes cuando se comparan los resultados metabólicos a largo plazo (control de las cifras tensionales, dislipemia y glicemia) de la cirugía (adrenalectomía) versus manejo conservador (observación y tratamiento médico), así como la relación de costos, los mismos son ampliamente favorables para el grupo de tratamiento quirúrgico, si es realizado por un equipo con experiencia, con cifras bajas de morbimortalidad ${ }^{(13)}$. Por lo que si bien no existe evidencia fuerte todavía como para realizar una recomendación formal, debería considerarse la resección de estas lesiones sobre todo en pacientes jóvenes, en quienes está previsto un seguimiento prolongado. El otro punto de discusión son los pacientes con lesiones menores de $4 \mathrm{~cm}$ pero que no tienen el aspecto típico de lesión benigna, o por lo menos no se puede establecer claramente la ausencia de malignidad en la tomografía. En estos casos puede ser de utilidad la realización de un PET-scan en búsqueda de hiperactividad metabólica según el SUV para definir la conducta final. Sin embargo, este paso del algoritmo de manejo del IS ha sido cuestionado recientemente, no solo en función del riesgo de que pase desapercibida una lesión maligna (que de hecho es bajo) cuyo pronóstico se sabe es desfavorable $^{(14)}$, sino de la relación costo/beneficio entre la suprarrenalectomía laparoscópica de entrada versus seguimiento prolongado. Existen trabajos recientes ${ }^{(15)}$ que muestran resultados a favor de la cirugía de entrada, debido, entre otros puntos, a elevadas tasas de indicación quirúrgica durante el seguimiento (hasta 44\%), sumado al impacto psicológico para el paciente de saberse portador de una lesión de significado incierto, y el de la acumulación de radiación ionizante. Los costos de la cirugía en comparación con la observación son levemente mayores al inicio, pero claramente menores cuando se comparan luego de un período de seguimiento prolongado.

Finalmente, el seguimiento de los IS no resecados constituye también un problema en la actualidad. Hasta el $90 \%$ de los IS unilaterales corresponde a pequeños adenomas no funcionantes, cuyo seguimiento mediante estudios de laboratorio e imagen tiene como objetivo principal la detección de cambios en su tamaño o la aparición de secreción hormonal, y con mucho menos frecuencia errores en el diagnóstico inicial. Con respecto al primer punto, el riesgo de transformación maligna de un adenoma no se conoce con precisión, no existiendo estudios de cohortes de calidad metodológica suficiente aún 
en cuanto a número de pacientes y tiempo de seguimiento. Sin embargo, se estima que el riesgo es casi nulo, y que apenas 7\%-10\% de los IS pueden aumentar de tama$\tilde{\text { ño }}{ }^{(16)}$. Aun si ese aumento de tamaño permanece por debajo del umbral de indicación quirúrgica, la misma podría surgir en caso de duplicar el tamaño inicial en un período breve de tiempo (un año). Por otra parte, el riesgo de aparición de secreción hormonal también es muy bajo. No hay reportes de aldosteronomas o feocromocitomas diagnosticados durante el seguimiento de IS inicialmente no secretantes. Sin embargo, existe un riesgo de $4 \%-12 \%$ a tres años de que se transforme en una lesión secretora de cortisol ${ }^{(17)}$, siendo el principal factor de riesgo el tamaño mayor a $3 \mathrm{~cm}$. Una vez definida la conducta no quirúrgica y por lo tanto observacional del IS, el protocolo de seguimiento que debe instaurarse constituye otro punto de controversia en función de la ausencia de evidencia científica de alto nivel que respalde las diferentes recomendaciones. La Sociedad Francesa de Endocrinología elaboró un consenso en el que propone realizar una tomografía computada a los seis meses para descartar aumento del tamaño, de mantenerse estable estará recomendada repetirla a los dos y cinco años. Para evaluar secreción hormonal se recomienda, además del examen clínico, repetir el test de supresión de dexametasona y metanefrinas en orina y plasma a los seis meses. De ser normales, solo repetir el test de dexametasona a los dos y cinco años ${ }^{(18)}$. Por otra parte, la Asociación Americana de Endocrinología y la Asociación Americana de Cirujanos Endócrinos proponen realizar la tomografía a los seis meses, al año y a los dos años con monitoreo hormonal anual durante $4-5$ años ${ }^{(19)}$. En caso de no existir aumento de tamaño o inicio de secreción hormonal, podría abandonarse el seguimiento en forma definitiva.

Finalmente debemos referirnos a un punto de capital importancia como es la resolución quirúrgica de patologías complejas además de poco frecuentes. Esto, sumado al problema demográfico de una población relativamente pequeña en nuestro país, lleva a que los cirujanos encuentren dificultades para alcanzar una curva de aprendizaje satisfactoria y por lo tanto a optimizar los resultados. Particularmente en el caso de la suprarrenalectomía laparoscópica se considera que la curva de aprendizaje se logra luego de 40 procedimientos del lado izquierdo y 30 a derecha ${ }^{(20)}$. Teniendo en cuenta un promedio de dos adrenalectomías por año de acuerdo a nuestros resultados, se entiende la dimensión del problema al que nos enfrentamos, y por ello creemos necesario concentrar dicha población de pacientes en aquel o aquellos centros donde un mismo equipo participe en la resolución de la patología endócrina compleja.

\section{Conclusiones}

La suprarrenalectomía laparoscópica es un procedimiento que permite resecar con seguridad la mayoría de las lesiones, que rara vez alcanzan un tamaño tal que impida de entrada el abordaje miniinvasivo. En nuestra serie fue la vía de abordaje factible en el $88 \%$ de los casos (14/16 pacientes), permitiendo a su vez una breve estadía posoperatoria y el alta precoz. Destacamos también en nuestra serie la baja morbilidad y la ausencia de mortalidad vinculadas al procedimiento, con cifras muy similares a las que fueran reportadas en otra serie histórica nacional ${ }^{(21)}$.

\section{Abstract}

Background: the incidence of adrenal incidentalomas has increased with the use of modern imaging techniques and surgical indication is based on suspected malignancy or hyperfunction.

Objective: to review a casuistry of surgically treated adrenal incidentalomas.

Method: 16 patients operated between 2010 and 2016 in two health care institutions. The study describes diagnostic aspects, operative techniques and morbimortality of the procedures.

Results: most of the procedures were performed by laparoscopic approach, 3 intraoperative complications arose, there being no deaths in the series. The most frequently encountered lesion was non-functioning adenoma.

Discussion: imaging constitutes the diagnostic key which results essential to make a decision as to whether to follow or operate an adrenal incidentaloma. Follow-up protocols are still under discussion, as is indication of surgery in subclinical functional lesions

Conclusions: laparoscopic adrenalectomy is a safe surgery, with morbidity and mortality rates which lie within the range described by relevant literature.

\section{Resumo}

Introdução: a incidência de incidentalomas suprarrenais aumentou com o uso de técnicas modernas de imagem e a indicação de cirurgia está baseada na suspeita de malignidade ou hiperfunção.

Objetivos: revisar una casuística de incidentalomas suprarrenais operados.

Pacientes e método: 16 pacientes operados no período 2010-2017 em duas instituições de assistência médica. Foram descritos os aspectos diagnósticos, técnica operatória e morbimortalidade dos procedimentos.

Resultados: a maioria dos procedimentos foram realizados por laparoscopia, registrando-se 3 complicações intraoperatórias, sem mortalidade na série. A les- 
ão mais frequentemente encontrada foi o adenoma não funcionante.

Discussão: os aspectos diagnósticos de um incidentaloma suprarrenal, fundamentais na tomada de decisão entre fazer um seguimento ou uma cirurgia, são as imagens. O protocolo de seguimento continua sendo causa de debate atualmente, como também a indicação de cirurgia nas lesões funcionantes subclínicas.

Conclusões: a suprarrenalectomia laparoscópica é uma cirurgia segura, com valores de morbimortalidade dentro dos intervalos descritos na literatura.

\section{Bibliografía}

1. Boland GW, Blake MA, Hahn PF, Mayo-Smith WW. Incidental adrenal lesions: principles, techniques, and algorithms for imaging characterization. Radiology 2008; 249(3):756-75.

2. Young WF Jr. Clinical practice: the incidentally discovered adrenal mass. N Engl J Med 2007; 356(6):601-10.

3. Barzon L, Sonino N, Fallo F, Palu G, Boscaro M. Prevalence and natural history of adrenal incidentalomas. Eur J Endocrinol 2003; 149(4):273-85.

4. Rodríguez G, Balboa O. Suprarrenalectomía videolaparoscópica. En: Rodríguez G, Balboa O, eds. Video cirugía, técnica quirúrgica. Montevideo: El Pais, 2003:183-92.

5. O'Neill CJ, Spence A, Logan B, Suliburk JW, Soon PS, Learoyd DL, et al. Adrenal incidentalomas: risk of adrenocortical carcinoma and clinical outcomes. J Surg Oncol 2010; 102(5):450-3.

6. Foti G, Faccioli N, Mantovani W, Malleo G, Manfredi R, Mucelli RP. Incidental adrenal lesions: Accuracy of quadriphasic contrast enhanced computed tomography in distinguishing adenomas from nonadenomas. Eur J Radiol 2012; 81(8):1742-50.

7. Ilias I, Sahdev A, Reznek RH, Grossman AB, Pacak K. The optimal imaging of adrenal tumours: a comparison of different methods. Endocr Relat Cancer 2007; 14(3):587-99.

8. Haider MA, Ghai S, Jhaveri K, Lockwood G. Chemical shift MR imaging of hyperattenuating $(>10 \mathrm{HU})$ adrenal masses: does it still have a role? Radiology 2004; 231(3):711-6.

9. Gust L, Taieb D, Beliard A, Barlier A, Morange I, de Micco C, et al. Preoperative 18F-FDG uptake is strongly correlated with malignancy, weiss score, and molecular markers of aggressiveness in adrenal cortical tumors. World J Surg 2012; 36(6):1406-10.
10. Evans PD, Miller CM, Marin D, Stinnett SS, Wong TZ, Paulson EK, et al. FDG-PET/CT characterization of adrenal nodules: diagnostic accuracy and interreader agreement using quantitative and qualitative methods. Acad Radiol 2013; 20(8):923-9.

11. Sancho JJ, Triponez F, Montet X, Sitges-Serra A. Surgical management of adrenal metastases. Langenbecks Arch Surg 2012; 397(2):179-94.

12. Barzon L, Fallo F, Sonino N, Boscaro M. Development of overt Cushing's syndrome in patients with adrenal incidentaloma. Eur J Endocrinol 2002; 146(1):61-6.

13. Toniato A, Merante-Boschin I, Opocher G, Pelizzo MR, Schiavi F, Ballotta E. Surgical versus conservative management for subclinical cushing syndrome in adrenal incidentalomas: a prospective randomized study. Ann Surg 2009; 249(3):388-91.

14. Bilimoria KY, Shen WT, Elaraj D, Bentrem DJ, Winchester DJ, Kebebew E, et al. Adrenocortical carcinoma in the United States: treatment utilization and prognostic factors. Cancer 2008; 113(11):3130-6.

15. Melck AL, Rosengart MR, Armstrong MJ, Stang MT, Carty SE, Yip L. Immediate laparoscopic adrenalectomy versus observation: cost evaluation for incidental adrenal lesions with atypical imaging characteristics. Am J Surg 2012; 204(4):462-7.

16. Tabarin A, Bardet S, Bertherat J, Dupas B, Chabre O, Hamoir E, et al. Exploration and management of adrenal incidentalomas: French Society of Endocrinology Consensus. Ann Endocrinol (Paris) 2008; 69(6):487-500.

17. Grumbach MM, Biller BM, Braunstein GD, Campbell KK, Carney JA, Godley PA, et al. Management of the clinically inapparent adrenal mass ("incidentaloma"). Ann Intern Med 2003; 138(5):424-9.

18. Mathonnet M, Peix JL, Sebag F. Chirurgie des Glandes Surrènales. Paris: Arnette, 2011.

19. Zeiger MA, Siegelman SS, Hamrahian AH. Medical and surgical evaluation and treatment of adrenal incidentalomas. J Clin Endocrinol Metab 2011; 96(7):2004-15.

20. Guerrieri M, Campagnacci R, De Sanctis A, Baldarelli M, Coletta M, Perretta S. The learning curve in laparoscopic adrenalectomy. Endocrinol Invest 2008; 31(6):531-6.

21. González D, Rodríguez G, Segura D, Balboa O, Ruso L. Adrenalectomía videolaparoscópica: experiencia inicial. Cir Endosc 2009; 10(3-4):113-7. 